\title{
Selenium ameliorates $S$. aureus-induced inflammation through ROS-mediated NLRP3 inflammasome in bovine mammary epithelial cells
}

Chongliang Bi ( lydxbcl@163.com )

Linyi University

Shujiu Zhang

Linyi University

He Tang

Linyi University

Hui Li

Linyi University

Research article

Keywords: Selenium, S. aureus, ROS, NLRP3

Posted Date: February 17th, 2020

DOI: https://doi.org/10.21203/rs.2.23719/v1

License: (c) (i) This work is licensed under a Creative Commons Attribution 4.0 International License.

Read Full License 


\section{Abstract}

Background Some research has indicated that selenium (Se) plays a significant role during mastitis. However the intracellular anti-inflammatory effect of Se is not fully clear. Due to the ability of Staphylococcus aureus ( $S$. aureus) to internalize into host cell, in this study we explored whether Se could regulate inflammation induced by $S$. aureus through reactive oxygen species (ROS)-mediated NLRP3 inflammasome in bMECs. Result bMECs were treated with $8 \mu \mathrm{mol} / \mathrm{L} \mathrm{Na} 2 \mathrm{SeO} 3$ for $12 \mathrm{~h}$ before infected with $S$. aureus for $2 \mathrm{~h}$. Through flow cytometry, Western blot and qPCR analysis, ROS and NLRP3 imflammasome were detected.

Result shown that the production of ROS was increased by S. aureus, Se exerted strong inhibitory effects on the production of ROS; The protein expression of NLRP3 inflammasome including NLRP3, ASC and Caspase-1 increased significantly after $S$. aureus infection, Se played an important role in regulating the expression of NLRP3, ASC and Caspase-1; To further investigate the anti-inflammatory effect of Se, the expression level of IL-1 $\beta$ associated molecule pro-IL-1 $\beta$ and IL-1 $\beta$ were detected. Result shown that the mRNA expression of IL-1 $\beta$ was up-regulated by $S$. aureus and after Se treatment the expression level of IL$1 \beta$ mRNA was markedly down-regulated, meanwhile Se play a regulation effect on the protein expression of Pro-IL-1 $\beta$ and IL-1 $\beta$.

Conclusions Here we show that ROS is involved in bMECs inflammation induced by $S$. aureus and Se ameliorates $S$. aureus -induced inflammation through ROS-mediated NLRP3 pathway in bMECs.

\section{Background}

Mastitis induced by Staphylococcus aureus (S. aureus) is a serious problem which will causes serious economic losses to the bovine industry $[1,2]$. Meanwhile due to the ability of $S$. aureus to internalize into host cell, mastitis induced by S. aureus is often difficult to cure. Along with the intensity and persistence of infection, most animals cannot escape the fate of elimination [3]. Thus finding effective ways to prevent or cure mastitis induced by $\mathrm{S}$. aureus has become research focus on dairy industry.

Innate immunity plays an important role in defensing against pathogens. Once stimulated innate immunity is triggered by pattern recognition receptors (PRRs) [4]. There are two PRRs mainly response for bacterial infection: Toll-like receptors (TLRs) and Nod-like receptors (NLRs) $[5,6]$. TLRs are located at the cell surface or within endosomal membranes. However NLRs mainly mediate cytosolic recognition of microbial molecules and promote their clearance [7]. Our previous study have already proved that TLRs play a crucial role in regulation inflammation induced by S. aureus in bMECs [8]. However the regulation effect of NLRs on S. aureus intracellular is still not clear, which challenge us for further detailed investigations and understanding.

Unlike TLR2, NLRs were capable to form inflammasomes in response to their specific stimulators $[9,10]$. Among them the most characterized member of the NLR family is the NLRP3 inflammasome [11]. Many studies have provided the activation process of NLRP3 pathway. Briefly, NIRP3 inflammasome composed 
of NLRP3, ASC and caspase-1, the activation of NLRP3 inflammasome ultimately process pro-IL-1 $\beta$ into IL-1 $\beta$. Moreover, induce inflammatory response $[12,13]$. Originally NLRP3 was hypothesized to be a cytosolic receptor. However with the deepening study, it seems highly improbable that NLRP3 acts as a receptor to directly bind to bacterial stimuli. ROS, produced by many known activators of NLRP3 inflammasomes, are shown to be a critical mechanism triggering NLRP3 inflammasome formation [14]. Furthermore S. aureus have been found to activate the NLRP3 inflammasome in monocytes/macrophages leading to inflammation [15]. However whether ROS offers a linked to the S. aureus infection and the antivation of NLRP3 inflammasome in bMECs is still unknown.

Se, an essential micronutrient, is a widely used feedstuff additive in dairy industry for a series of biology function such as antioxidant and immune anti-inflammation $[16,17]$. Some research has indicated that Se deficiency is associated with an increased incidence of placental retentions, metritis, mastitis, susceptibility to infections and reduced fertility [18]. On dairy industry, studies demonstrated adding Se in dietary could affect the innate and the adaptive immune responses to mastitis [19]. The underlying mechanisms of Se in regulating mastitis is complicated. Our previous studies have confirmed that $\mathrm{Se}$ could ameliorates $\mathrm{S}$. aureus-induced inflammation in bovine mammary epithelial cells by inhibiting activation of TLR2 signaling pathways. However further research is needed to determine whether NLRP3 pathway is involved in mastitis induced by S. aureus and whether Se is involved in the regulation of NLRP3 pathway in bMECs.

\section{Methods}

\section{Culture of primary bMECs and treatment}

bMECs were isolated from the udders of dairy cows as previously described in Dulbecco's modified Eagle's medium (DMEM/F12; Gibco, US) [8], and 10\% heat-inactivated fetal bovine serum (Gibco, Grand island), $5 \mu \mathrm{g} / \mathrm{mL}$ insulin (Sigma, US), $1 \mu \mathrm{g} / \mathrm{mL}$ hydrocortisone (Sigma, US) and $10 \mathrm{ng} / \mathrm{mL}$ epidermal growth factor (Sigma, US) were added to the DMEM. Sodium selenite was diluted in DMEM/F12 medium, bMECs were treated with Se for $12 \mathrm{~h}$ before S. aureus infection. S. aureus (ATCC29213, US) was added to the 6 -well plates at $\mathrm{MOI}=1: 1$ for $2 \mathrm{~h}$. All cells were washed with serum-free medium and serum starved for $1 \mathrm{~h}$ for further experiments.

\section{Flow Cytometer analysis}

$3 \mu \mathrm{M}$ DHR123 ( Dihydrorhodamine 123; Sigma, US ) was added into cell culture medium $3 \mathrm{~h}$ before harvest. Cells were collected with cold PBS and Flow Cytometer (BD Accuri ${ }^{\text {TM }}$ C6, US) was used to analysis ROS level in bMECs cells.

\section{Western blot analysis}

Total protein was extracted by bicinchoninic acid protein assay kit (BioChain, US) according to the manufacturer's instructions. The proteins were loaded onto a $10 \%$ sodium dodecyl sulphate polyacrylamide gel for electrophoresis and transferred to PVDF membranes (Millipore, Germany). The 
membranes were blocked in $5 \%$ nonfat milk for $1 \mathrm{~h}$ to $2 \mathrm{~h}$ and incubated overnight with the primary antibodies, including NLRP3, ASC, Caspase-1 and IL-1 $\beta$ (CST, US). Then, all of the membranes were subsequently incubated with HRP-conjugated goat anti-rabbit antibodies (CST, US). The blots were washed with PBS-T and processed with supersignal west pico chemiluminescent substrate.

\section{Quantitative Real-Time PCR analysis}

The total RNA were extracted according to the manufacturer's instructions using Trizol reagent (Invitrogen, US). The quantity and quality of the extracted RNA were evaluated with a Nanodrop 2000 spectrophotometer (Thermo, US). RNA was converted to cDNA using a reverse transcriptase synthesis kit (Takara, Japan). The PCR reaction system contained $10 \mu \mathrm{L}$ of SYBR Green PCR mix, $0.8 \mu \mathrm{L}$ of each primer (both $10 \mu \mathrm{mol} / \mathrm{L}$ ), and $2 \mu \mathrm{L}$ of cDNA template in a final volume of $20 \mu \mathrm{L}$ per reaction (Takara, Japan). The sequences of the primers are as follow:

Table 1

primers used in the experiment

\begin{tabular}{|c|c|}
\hline Gene & Sequences \\
\hline$\beta$-actin & $\begin{array}{l}\text { F: ACATCCGCAAGGACCTCTA } \\
\text { R: CCATGCCAATCTCATCTCGTT }\end{array}$ \\
\hline IL-1 $\beta$ & $\begin{array}{l}\text { F: GCTATGAGCCACTTCGTGAGGAC } \\
\text { R: GATTGAGGGCGTCGTTCAGGAT }\end{array}$ \\
\hline
\end{tabular}

\section{Statistical Analysis}

All data are shown as the mean \pm S.E.M, One-way ANOVA and Dennett's test were used for statistical analyses. Values of $\mathrm{P}<0.05$ were considered statistically significant.

\section{Result}

\section{Change in ROS level}

The result are shown in Fig. 1. Compared with CG (bMECs without any treatment, bMECs, Fig. 1A) and SeG (bMECs treated with Se for $12 \mathrm{~h}$, bMECs + DHR123 + Se, Fig. 1C), the production level of ROS increased significantly in MG (bMECS infected with S. aureus for $2 \mathrm{~h}$, bMECs + DHR123 + S. aureus, Fig. 1D); Compared with MG the production level of ROS was markedly decreased in TG (bMECs treated with Se for $12 \mathrm{~h}$ before infected with S. aureus for $2 \mathrm{~h}$, bMECs + DHR123 + Se + S. aureus, Fig. 1E).

\section{Change in NLRP3 inflammasome}

As shown in Fig. 2. Compared with CG and SeG the expression of NLRP3 was significantly increased in MG and compared with MG the NLRP3 expression level decreased significantly in TG (Fig. 2A and B); S. aureus infection markedly increased the expression of ASC and Se treatment significantly decreased the 
ASC protein expression (Fig. 2A and C); Compared with CG and SeG, the expression level of Pro-caspase1 and Caspase- 1 were both up-regulated by $S$. aureus and Se could down-regulated the expression level of Pro-caspase-1 and Caspase-1 significantly (Fig. 2A, D and E).

\section{Changes in the expression of IL-1 $\beta$}

Result shown that the IL-1 $\beta$ mRNA expression increased significantly in MG, and compared with $M G$, the expression of IL-1 $\beta$ mRNA was decreased significantly in TG (Fig. 3C); Compared with CG and SeG the protein expression level of Pro-IL-1 $\beta$ was up-regulated by $S$. aureus in MG, Se exerted strong inhibitory effects on the expression of Pro-IL-1 $\beta$ (Fig. 3A and B); Compared with CG and SeG, the production of IL-1 $\beta$ increased significantly induced by S. aureus and this effect was blocked by Se in TG (Fig. 3A and D).

\section{Discussion}

Oxidative Stress (OS) is caused by an imbalance between free radical formation and antioxidant defense [20]. And OS induces production of ROS in cells [21]. Due to mastitis itself presents an OS, in this study we first detected the ROS level to understand the impact of S. aureus on bMECs OS. Result shown that the bMECs ROS level increased significantly after S. aureus infection, and Se obviously suppressed the production of ROS induced by S.aureus. The result demonstrated that ROS is involved in bMECs inflammation induced by S. aureus and also Se is involved in regulation the production of ROS in bMECs.

The activation of NLRP3 inflammasomes has been associate with a serious of pathological conditions including mastitis [22, 23]. NLRP3 inflammasomes is a tripartite protein that consists of NLRP3, ASC and caspase-1. The NLRP3 protein interacts with ASC to initiate inflammasome assembly [24]. And caspase-1 is mainly responsible for the maturation of inactive cytokine precursors such as IL-1 $\beta$, a powerful

inflammatory mediator, which is one of the most studied cytokines related to the innate immune response [25]. NLRP3 inflammasome activation in response to a several of signals, such as virus, S. aureus and Escherichia coli $[26,27]$. It seems that a broad range of stimuli have been demonstrated to cause NLRP3 activation, however research shown that ROS serves as a triggering factor to activate NLRP3 inflammasomes [28]. Flow cytometry analysis showed that Se play a regulation effect on the production of ROS induced by S. aureus. Therefore we detected the effect of Se on NLRP3 pathway subsequently. Result shown that Se suppressed the expression of NLRP3, ASC and caspase-1, which indicating an inhibitory effect of NLRP3 inflammasome; meanwhile the expression of pro-IL-1 $\beta$ and IL-1 $\beta$ were also suppressed, which indicating that Se could protect bMECs from $S$. aureus injury.

In conclusion, this study proved the protective effect of Se on S. aureus-induced inflammation in bMECs. This effect was at least partly achieved by the blocking ROS-mediated NLRP3 inflammasome. This result provide a complement to the research on anti-inflammatory effect of Se.

\section{Abbreviations}


bMECS: Bovine mammary epithelial cells; ROS: Reactive oxygen species; S. aureus: Staphylococcus aureus; DHR123: Dihydrorhodamine 123; OS: Oxidative Stress.

\section{Declarations}

\section{Funding}

The investigation was supported by the National Natural Science Foundation of China (NO. 31802254).

\section{Availability of date and materials}

All data used during the study appear in the submitted article.

\section{Authors' contributions}

$\mathrm{Bi} \mathrm{CL}$ contributed to the overall study design and supervised all research. $\mathrm{Li} \mathrm{H}$ analyzed the data and Zhang SJ prepared Figures and contributed partly to writing and finally revising the manuscript and data analysis. Tang $\mathrm{H}$ drafted and revised the first version of the manuscript. All the authors reviewed and finally approved the manuscript.

\section{Ethical approval}

The protocol was approved by the Animal Care and Ethics Committee of Linyi University.

\section{Competing interests}

The authors declare that they have no conflict of interest.

\section{Acknowledgement}

Not Applicable.

\section{References}

1. Rahmeto $A$, Hagere $H$, Mesele $A$ et al. Bovine mastitis: prevalence, risk factors and isolation of Staphylococcus aureus in dairy herds at Hawassa milk shed, South Ethiopia. BMC Vet Res. 2016; 12: 270.

2. Pumipuntu N, Kulpeanprasit S, Santajit S et al. Screening method for Staphylococcus aureus identification in subclinical bovine mastitis from dairy farms. Vet World. 2017; 10: 721-6.

3. Sowash MG, Uhlemann AC. Community-associated methicillin-resistant Staphylococcus aureus case studies. Methods Mol. Biol. 2013; 1085: 25-69.

4. Jeong YJ, Kang MJ, Lee SJ et al. NOD2 and RIP2 contribute to innate immune responses in mouse neutrophils. Immunology. 2014; 143: 269-76. 
5. Philpott DJ, Girardin SE. The role of Toll-like receptors and Nod proteins in bacterial infection. Mol Immunol. 2004; 41: 1099-108.

6. Philpott DJ, Sorbara MT, Robertson SJ et al. NOD proteins: regulators of inflammation in health and disease. Nat Rev Immunol. 2014; 14: 9-23.

7. Girardin SE, Travassos LH, Herve M et al. Peptidoglycan molecular requirements allowing detection by NOD1 and NOD2. J Biol Chem. 2003; 278:41702-8.

8. Wang $\mathrm{H}, \mathrm{Bi} \mathrm{CL}$, Wang YJ et al. Selenium ameliorates Staphylococcus aureus-induced inflammation in bovine mammary epithelial cells by inhibiting activation of TLR2, NF-KB and MAPK signaling pathways. BMC Vet Res. 2018; 14: 197.

9. Jason HM, David BAJ, Ashley LD et al. Staphylococcus aureus Leukocidin A/B(LukAB) kills human monocytes via host NLRP3 and ASC when extracellular, but not intracellular. Plos Pathog. 2015; 11(6): e1004970.

10. Zhao XB, Pu DB, Zhao ZZ et al. Teuvincenone F suppresses LPS-induced inflammation and NLRP3 Inflammasome activation by attenuating NEMO ubiquitination. Front Pharmacol. 2017; 8: 565.

11. Duewell $\mathrm{P}$, Kono $\mathrm{H}$, Rayner KJ et al. NLRP3 inflammasome are required for atherogenesis and activated by cholesterol crystals. Nature. 2010; 464: 1357-1361.

12. Lee DJ, Du F, Chen SW et al. Regulation and function of the caspase-1 in an inflammatory microenvironment. J Invest Dermatol. 2015; 135(8): 2012-2020.

13. Li YF, Gayani N, Sun Y et al. Analyses of caspase-1-regulated transcriptomes in various tissues lead to identification of novel IL-1 $\beta$-, IL-8- and sirtuin-1-independent pathway. J Hematol Oncol. 2017; 10: 40.

14. Justine MA, Xia M, Zhang Y et al. Redox Regulation of NLRP3 Inflammasomes: ROS as Trigger or Effector? Antioxid Redox Signal. 2015; 22(13): 1111-1129.

15. Kebaier C, Chamberland RR, Allen IC et al. Staphylococcus aureus alpha-hemolysin mediates virulence in a murine model of severe pneumonia through activation of the NLRP3 inflammasome. $J$ Infect Dis. 2012; 205: 807-817.

16. Smith AD, Cheung $L$, Beshah $E$ et al. Selenium status alters the immune response and expulsion of adult Heligmosomoides bakeri worms in mice. Infect Immun 2013; 81:2546-2553.

17. Youcef $M$, Isabelle D. Selenium in cattle: A review. Molecules. 2016; 21(3): 545.

18. Bozena H, Marta K, Sylvie $\mathrm{S}$ et al. A summary of new findings on the biological effects of selenium in selected animal species-A critical review. Int J Mol Sci. 2017; 18(10): 2209.

19. Yang $\mathrm{H}$, Fang J, Jia $X$ et al. Chemopreventive effects of early-stage and late-stage supplementation of vitamin $E$ and selenium on esophageal carcinogenesis in rats maintained on a low vitamin E/selenium diet. Carcinogenesis. 2011; 32: 381-388.

20. Hu XQ, Song R, Zhang LB. Effect of Oxidative Stress on the Estrogen-NOS-NO-KCa Channel Pathway in Uteroplacental Dysfunction: It's Implication in Pregnancy Complications. Oxid Med Cell Longev. 2019; 2019: 9194269. 
21. Kondoh $\mathrm{M}$, Ohga $\mathrm{N}$, Akiyama $\mathrm{K}$ et al. Hypoxia-induced reactive oxygen species cause chromosomal abnormalities in endothelial cells in the tumor microenvironment. PLoS One. 2013; 8(11): e80349.

22. Benko S, Philpott DJ, Girardin SE et al. The microbial and danger signals that activate Nod-like receptors. Cytokine. 2008; 43(3):368-73.

23. Ma JK, Zhu S, Guo YF et al. Selenium attenuates Staphylococcus aureus mastitis in mice by inhibiting the activation of NLRP3 inflammasome and NF-KB/MAPK pathway. Biol Trace Elem Res. 2019; 191: 159-166.

24. Nathan K, Devon J, Duan YH et al. The NLRP3 Inflammasome: An Overview of Mechanisms of Activation and Regulation. Int J Mol Sci. 2019; 20(13): 3328.

25. Dinarello CA. Immunological and inflammatory functions of the interleukin-1 family. Annu Rev Immunol. 2009; 27:519-50.

26. Ichinohe T, Lee HK, Ogura $Y$ et al. Inflammasome recognition of influenza virus is essential for adaptive immune responses. J Exp Med. 2009.16; 206(1):79-87.

27. Muruve DA, Pétrilli V, Zaiss AK et al. The inflammasome recognizes cytosolic microbial and host DNA and triggers an innate immune response. Nature. 2008.6; 452(7183):103-7.

28. Ma MW, Wang J, Dhandapani KM et al. NADPH Oxidase 2 Regulates NLRP3 Inflammasome Activation in the Brain after Traumatic Brain Injury. Oxid Med Cell Longev. 2017; 2017:6057609.

\section{Figures}



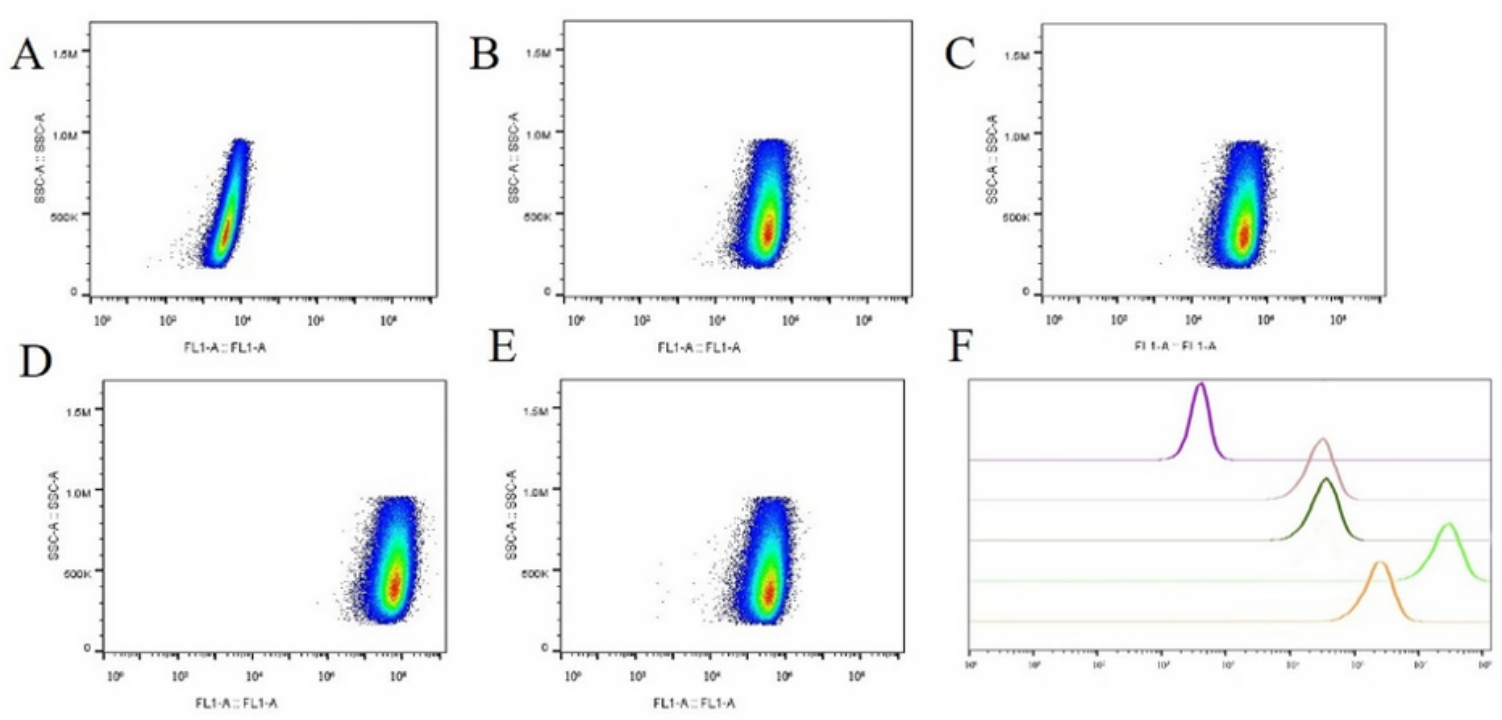

bMEC

bMECs+ DHR123

bMECs+ DHR123+Se

bMECs+ DHR123+S, aureus

bMECs+ DHR123+Se+S. aureus

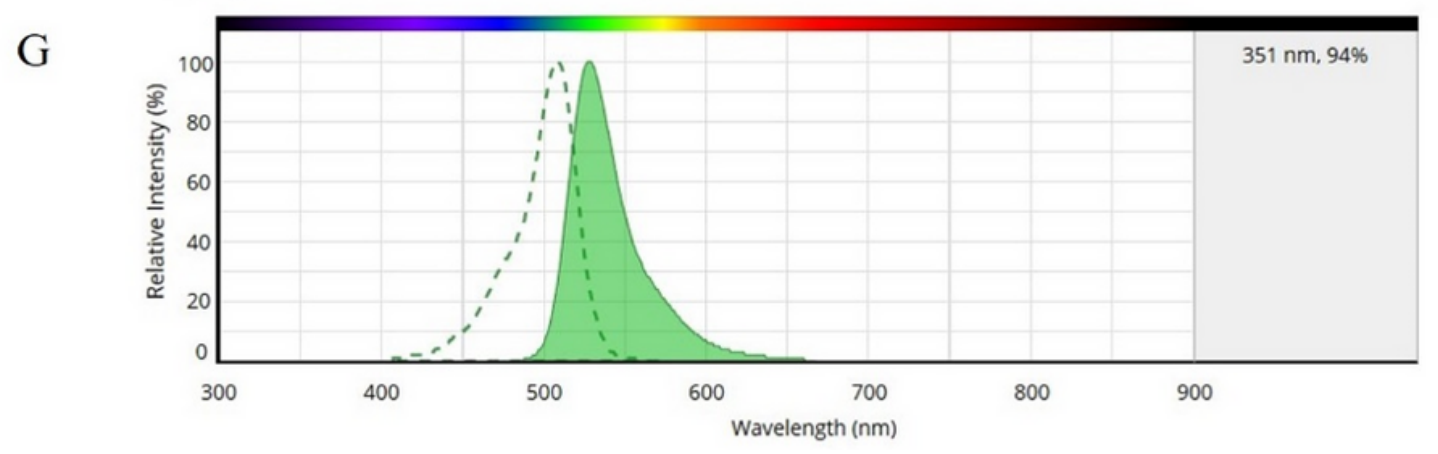

Figure 1

The ROS level detected by Flow Cytometer analysis; A: bMECs; B: bMECs+DHR123; C:

bMECs+DHR123+Se; D: bMECs+DHR123+S. aureus; E: bMECs+DHR+Se+S. aureus; F: The analysis of the intensity peaks; G: Relative intensity of different wavelength 
A

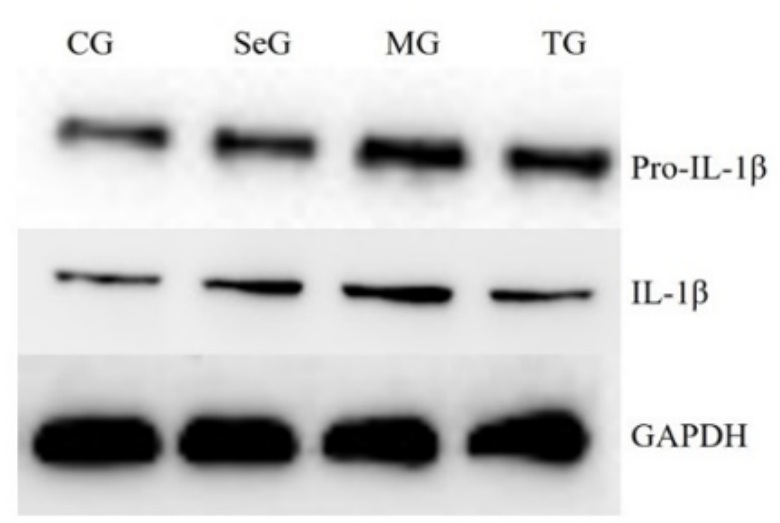

$\mathrm{C}$

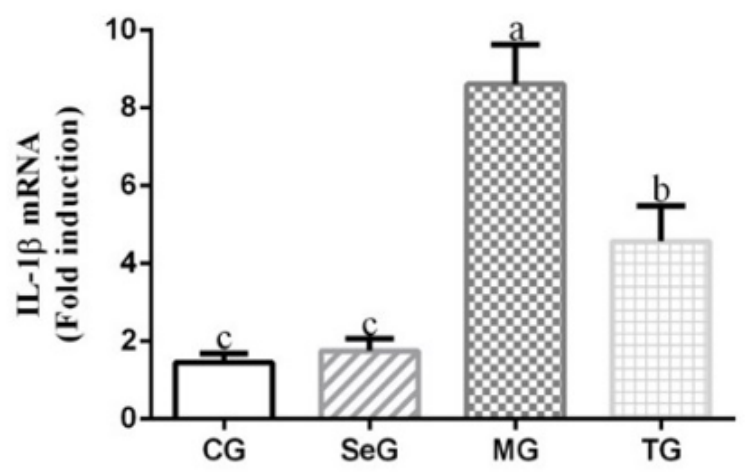

$\mathrm{B}$

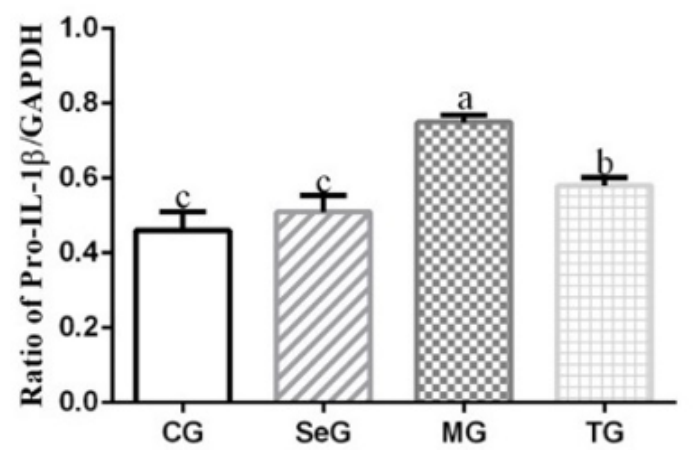

$\mathrm{D}$

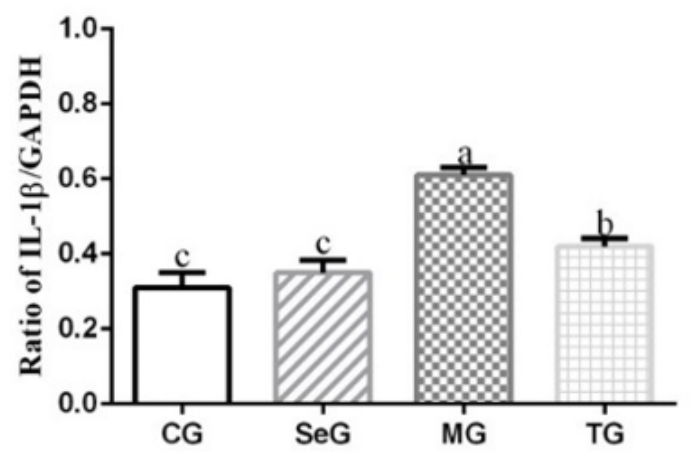

Figure 2

The effect of Se on the expression level of NLRP3 inflammasome. A: West blot analysis; B The expression level of NLRP3 protein; C: The expression level of ASC protein; D: The expression level of Procaspase-1 protein; E: The expression level of Caspase-1 protein Notes: CG: bMECs without any treat; SeG: bMECs treated with Se without infection; MG: bMECs infected with S. aureus without treated with Se; TG: bMECs treated with Se and infected with $S$. aureus. 


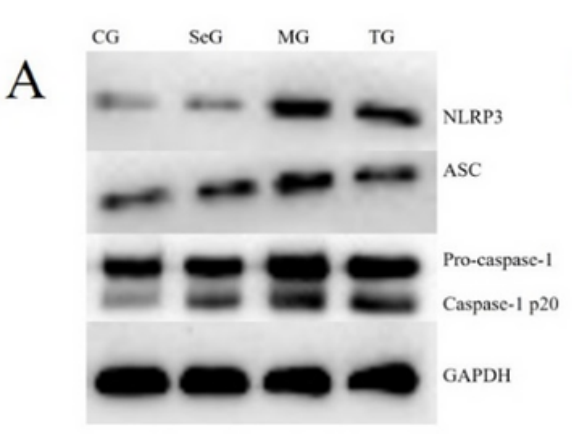

$\mathrm{D}$

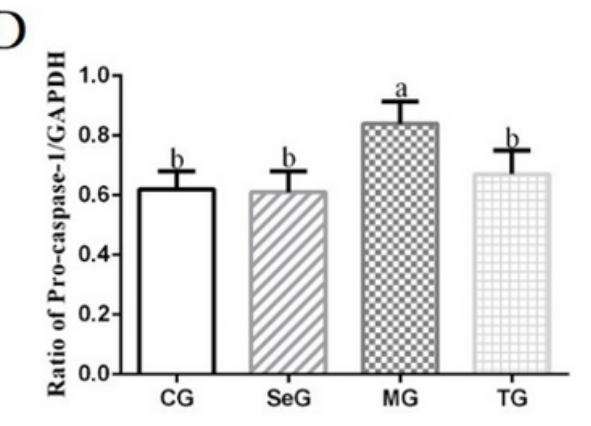

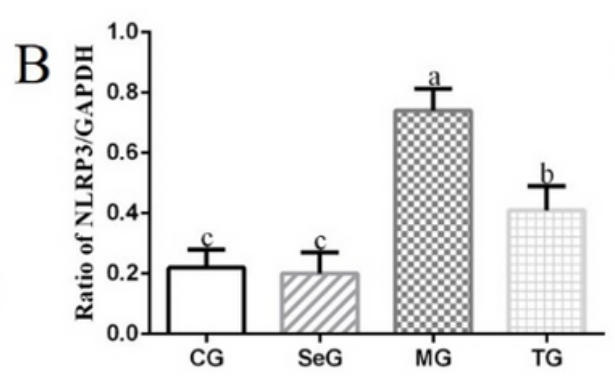

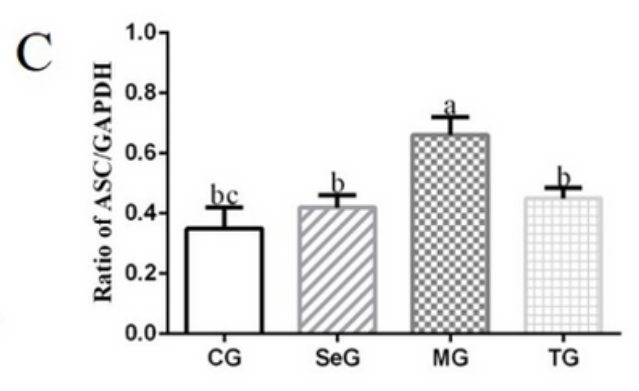

E

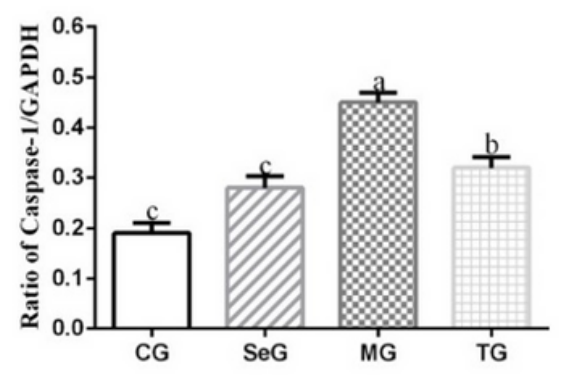

\section{Figure 3}

The effect of Se on the expression level of IL-1 $\beta$. A: Western blot analysis; B: The expression level of ProIL-1 $\beta$ protein; $C$ : The mRNA expression of IL-1 $\beta$; $D$ : The expression level of IL-1 $\beta$ protein. 